\title{
A Retro-Directive Antenna Array With Phase Conjugation Circuit Using Subharmonically Injection-Locked Self-Oscillating Mixers
}

\author{
Shih-Chieh Yen, Student Member, IEEE, and Tah-Hsiung Chu, Member, IEEE
}

\begin{abstract}
In this paper, a novel phase conjugation circuit and its application to retro-directive antennas are presented. The phase conjugation circuit uses a balanced circuit structure with subharmonically injection locked self-oscillating mixers oscillating at $\omega$. In the operation, an input signal at $\omega / 2$ is converted to its conjugated signal with no external source required for LO signal pumping, and the output signal frequency is locked at the same frequency of the input signal. The developed phase conjugation circuit is implemented with active antennas to become a retrodirective antenna array. Both the theoretical and measured results of phase conjugation and retro-directive performance are shown in good agreement.
\end{abstract}

Index Terms-Injection locked oscillators, phase conjugation, retro-directive antennas.

\section{INTRODUCTION}

A RETRO-DIRECTIVE antenna has the unique characteristic that it retransmits incident signals backward in the direction of the sources. Although the direction of arrival techniques may have this performance [1], [2], they may require complicated digital signal processing, giving restrictions on high-frequency and high-speed applications. The main feature of a retro-directive antenna is to have its retransmitted signal and incident signal to be a phase conjugation pair at the microwave frequency. It can find applications [3]-[7] in aircraft carrier landing system, automatic tolls, cargo tracking, collision avoidance, intelligent cruise control, and channel markers.

To illustrate the basic concept of phase conjugation, an incident wave $\bar{E}_{i}$ is expressed as

$$
\bar{E}_{i}=\bar{A}(\bar{r}) e^{j[\omega t+\phi(\bar{r})]}
$$

where $\bar{r}$ is a position vector, $\omega$ is the angular frequency, and $\phi(\bar{r})$ is the position-dependent phase term. Its phase-conjugated wave becomes

$$
\bar{E}_{r}=\bar{A}(\bar{r}) e^{j[\omega t-\phi(\bar{r})]} .
$$

This indicates that the incident wave and its phase-conjugated wave have the same wavefronts but propagate in opposite directions, provided that the environment is reciprocal.

Manuscript received July 25, 2002; revised December 31, 2002. This work was supported in part by the National Science Council of Taiwan, R.O.C., under Grants 91-2219-E-002-030 and 91-2219-E-002-018, and in part by the Ministry of Education of Taiwan, R.O.C., under Grant 89-E-FA06-2-4-6.

The authors are with the Graduate Institute of Communication Engineering, National Taiwan University, Taipei, Taiwan, R.O.C. (e-mail: d88003@ew.ee.ntu.edu.tw; thc@ew.ee.ntu.edu.tw).

Digital Object Identifier 10.1109/TAP.2003.822436

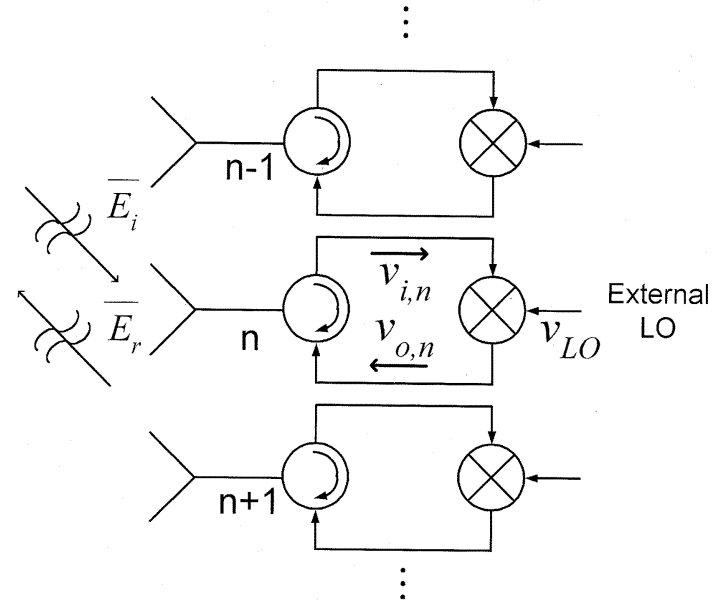

Fig. 1. Retro-directive antenna array using heterodyne array structure.

One well-known retro-directive antenna structure is a heterodyne array [5], [8], as shown in Fig. 1. For the $n$th element, if the incident signal to the mixer is

$$
\nu_{i, n}=A_{n} e^{j\left(\omega t+\phi_{n}\right)}
$$

and the external LO signal is at the double frequency $2 \omega$ as

$$
\nu_{\mathrm{LO}}=A e^{j(2 \omega t+\theta)}
$$

where $A$ and $\theta$ are its amplitude and phase angle. The mixer output signal is then proportional to the conjugation of the incident signal with $-\phi_{n}$ phase term as

$$
\nu_{o, n}=c \nu_{i, n} \nu_{\mathrm{LO}}=c A_{n} A e^{j\left(\omega t+\theta-\phi_{n}\right)}=c A e^{j \theta} \nu_{i, n}^{*}
$$

where $c$ is a constant to account for the mixing product. Therefore, as the LO signal is pumped in equal amplitude and synchronized phase for each element, the retransmitted wave become phase conjugated to the incident wave.

This heterodyne array approach has been popularly applied in the areas of millimeter-wave and quasi-optics; however, it may have the following limitations. First, an external LO pumping signal is required. Secondly, it only allows single frequency operation, because the output signal frequency changes in the opposite direction of the input signal frequency. Thirdly, its mixer needs to have good isolation between incident signal and output signal, since they are at the same frequency.

In this paper, a novel phase conjugation circuit is presented to solve these limitations. Using a balanced circuit structure with subharmonically injection locked self-oscillating mixers (SILSOMs), no external LO source is required and the output 


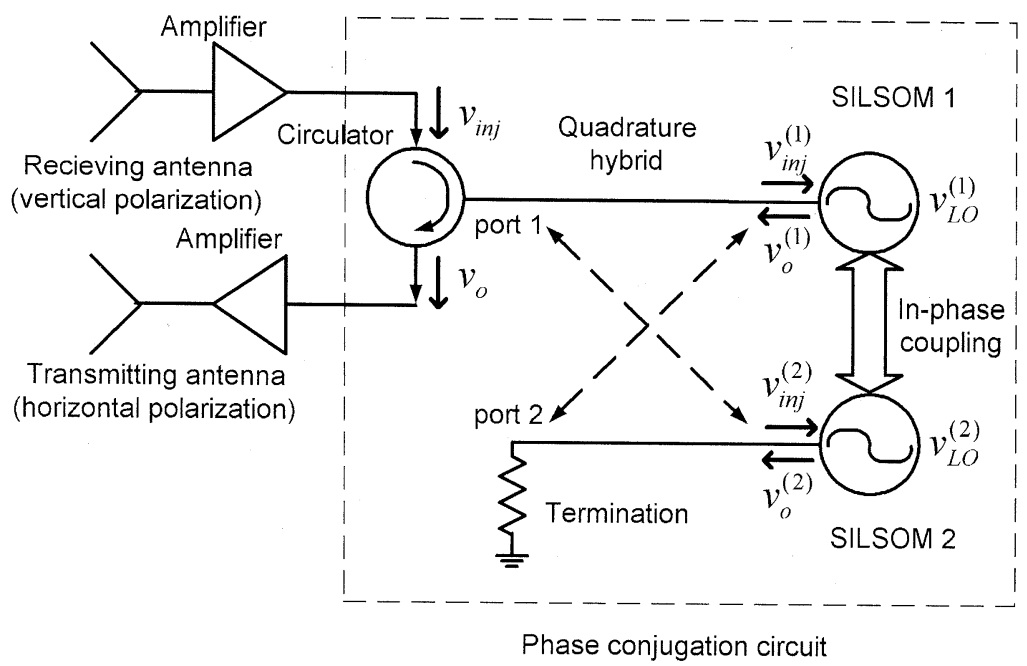

Fig. 2. Block diagram of a basic retro-directive antenna element.

signal is locked at the same frequency as the input signal. With the oscillator locking characteristics, frequency modulation operation is also possible. A retro-directive antenna array is then designed by loading the proposed phase conjugation circuit with active antennas. Measurements of the bistatic retransmitted radiation pattern are conducted to illustrate its retro-directive characteristics.

Section II describes the design principles and basic formulations. Details of circuit implementation are given in Section III. In Section IV, numerical and experimental results of a two-element phase conjugation circuit and retro-directive antenna array are presented. Conclusions are given in Section V.

\section{DESIGn AND Formulations}

\section{A. Design Principles}

The proposed retro-directive antenna array basically uses the heterodyne array concept but with a novel design on the phase conjugation circuit to tackle the limitations of heterodyne array described in the previous section. The basic components of the phase conjugation circuit are mutually coupled subharmonically injection-locked oscillators (SILOs) [9]. As the oscillator is well biased, the SILO also becomes a mixer itself due to the transistor nonlinear effect. In other words, the oscillator behaves as a SILSOM. By using a balanced circuit structure with SILSOMs having in-phase coupling at the fundamental frequency, good isolation between the incident and output signals can be achieved.

Fig. 2 shows the basic block diagram of a retro-directive antenna element. It is constructed by using a quadrature hybrid with two SILSOMs coupled in-phase at the fundamental oscillating frequency $\omega[10]$. This then forces two SILSOMs to oscillate coherently. The LO oscillating signals are given as

$$
\nu_{\mathrm{LO}}^{(1)}=\nu_{\mathrm{LO}}^{(2)}=A_{\mathrm{LO}} e^{j(\omega t+\theta)}
$$

where the superscripts (1) and (2) are used to specify the two SILSOMs.

As an input signal at the subharmonic frequency $\omega / 2$

$$
\nu_{\mathrm{inj}}=A_{\mathrm{inj}} e^{j(\omega t / 2+\phi)}
$$

is injected through a quadrature hybrid, the signals to the SILSOMs become

$$
\begin{aligned}
& \nu_{\text {inj }}^{(1)}=\frac{A_{\text {inj }}}{\sqrt{2}} e^{j(\omega t / 2+\phi-\pi / 2)} \\
& \nu_{\text {inj }}^{(2)}=\frac{A_{\text {inj }}}{\sqrt{2}} e^{j(\omega t / 2+\phi-\pi)} .
\end{aligned}
$$

These two signals are then mixed with LO signals to give the output signals

$$
\begin{aligned}
\nu_{o}^{(1)}= & c_{1} \frac{A_{\text {inj }}}{\sqrt{2}} e^{j(\omega t / 2+\phi-\pi / 2)} \\
& +c_{2} \frac{A_{\text {inj }}}{\sqrt{2}} A_{\mathrm{LO}} e^{j(\omega t / 2+\theta-\phi+\pi / 2)}+\text { h.o.t. } \\
\nu_{o}^{(2)}= & c_{1} \frac{A_{\text {inj }}}{\sqrt{2}} e^{j(\omega t / 2+\phi-\pi)} \\
& +c_{2} \frac{A_{\text {inj }}}{\sqrt{2}} A_{\mathrm{LO}} e^{j(\omega t / 2+\theta-\phi+\pi)}+\text { h.o.t. }
\end{aligned}
$$

where $c_{1}$ and $c_{2}$ are coefficients of the first-order and the second-order responses, respectively, and "h.o.t." accounts for the higher order terms.

Therefore, the output signals at port 1 and port 2 of the quadrature hybrid are

$$
\begin{aligned}
& \nu_{o, \text { port1 }}=c_{2} A_{\mathrm{inj}} A_{\mathrm{LO}} e^{j(\omega t / 2+\theta-\phi)}+\text { h.o.t. } \\
& \nu_{o, \text { port2 }}=c_{1} A_{\mathrm{inj}} e^{j(\omega t / 2+\phi-3 \pi / 2)}+\text { h.o.t. }
\end{aligned}
$$

respectively. Here, the separation of two signals $\nu_{o, p o r t 1}$ and $\nu_{o \text {,port2 }}$ at the same frequency is achieved by using the balanced circuit structure. In other words, the dominant odd-order terms (the first-order terms) are terminated at port 2, whereas the dominant even-order terms (the second-order terms) contribute to the output signal as

$$
\nu_{o}=\nu_{o, \text { port } 1} \cong c_{2} A_{\mathrm{LO}} e^{j \theta} \nu_{\mathrm{inj}}^{*}
$$

In (11), the output signal is proportional to the phase conjugation of the input signal and $\left|c_{2} A_{\mathrm{LO}}\right|$ is the conversion gain of the phase conjugation circuit. Note, (10a) and (10b) show that the good isolation of the input signal $\nu_{\mathrm{inj}}$ at the output (or port 2) depends on the balanced structure of quadrature hybrid. 


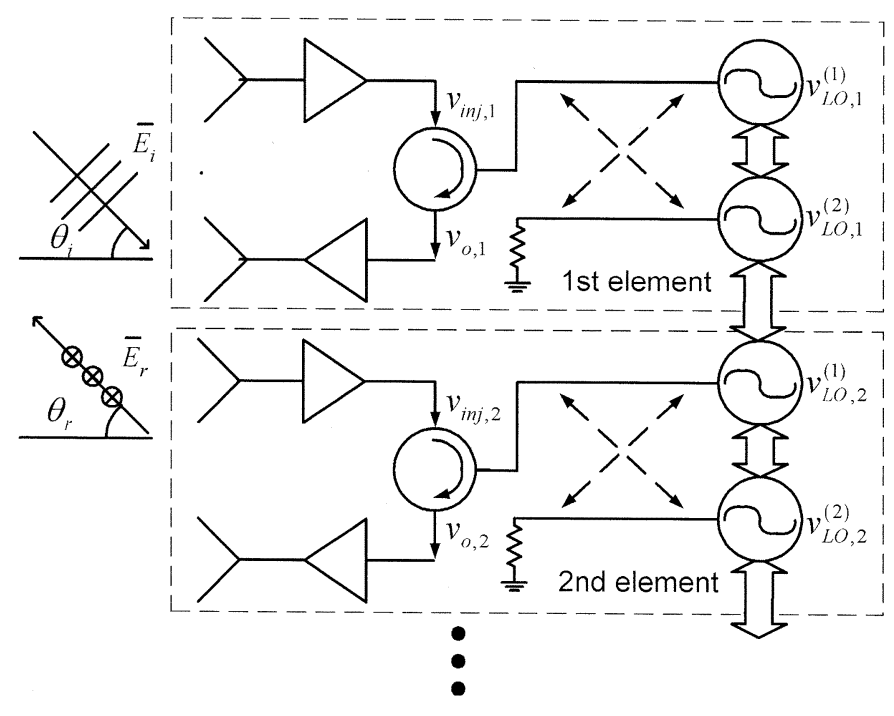

Fig. 3. Block diagram of a 1-D retro-directive antenna array.

Loading a receiving antenna and a transmitting antenna with amplifiers at port 1 through a circulator, a basic retro-directive antenna element is constructed as shown in Fig. 2. The receiving and the transmitting antennas are arranged in orthogonal polarizations to have a better isolation of input and output signals.

One can then extend the proposed structure as a one-dimensional (1-D) retro-directive antenna array shown in Fig. 3 with all the SILSOMs coupled in-phase. For an $N$-element retro-directive antenna array, the output signals are given as

$$
\left[\begin{array}{c}
\nu_{o, 1} \\
\nu_{o, 2} \\
\vdots \\
\nu_{o, N}
\end{array}\right]=c_{2} A_{\mathrm{LO}} e^{j \theta}\left[\begin{array}{c}
\nu_{\mathrm{inj}, 1}^{*} \\
\nu_{\mathrm{inj}, 2}^{*} \\
\vdots \\
\nu_{\mathrm{inj}, N}^{*}
\end{array}\right] .
$$

As shown in Fig. 3, an incident wave from the incident angle $\theta_{i}$ with vertical polarization can be retransmitted back to the original direction $\theta_{r}=\theta_{i}$ with horizontal polarization.

\section{B. Practical Considerations}

The design equations given in (6)-(12) are derived under ideal situations. In practice, as the in-phase coupled oscillators are locked by injection signals, the oscillators are not in the same phase as (6) but with relative phase values among them. From [11]-[14], an injection-locked oscillator can be described by Adler's equation as

$$
\frac{d \theta_{o}}{d t}=\Delta \omega_{o}-\frac{\varepsilon \omega_{o}}{2 Q} \frac{A_{\mathrm{inj}}}{A_{o}} \sin \left(\theta_{o}-\theta_{\mathrm{inj}}\right)
$$

where $Q$ is the quality factor and $\omega_{o}$ is the oscillator free-running frequency. $A_{o}$ and $\theta_{o}$ are the amplitude and the phase of the oscillator output signal. $A_{\text {inj }}$ and $\theta_{\text {inj }}$ are the amplitude and the phase of the injection signal. The frequency detuning is $\Delta \omega_{o}=$ $\omega_{o}-\omega_{\text {inj }}$, and $\varepsilon$ is the coupling coefficient of the injection signal to the output signal.
In the following, a two-element retro-directive antenna array is taken as an example for the simplicity of illustration. The injection signals for each element are

$$
\begin{aligned}
& \nu_{\mathrm{inj}, 1}=A_{\mathrm{inj}} e^{j(\omega t / 2+\phi)} \\
& \nu_{\mathrm{inj}, 2}=A_{\mathrm{inj}} e^{j(\omega t / 2+\phi-\xi)}
\end{aligned}
$$

with a phase difference $\xi$. The four subharmonically injectionlocked oscillators are under the nearest neighbor coupling condition; hence their phases should satisfy

$$
\begin{aligned}
\frac{d \theta_{1}^{(1)}}{d t}=\Delta \omega_{1}^{(1)} & -\frac{\omega_{1}^{(1)}}{2 Q}\left[\frac{\varepsilon_{c} A_{1}^{(2)}}{A_{1}^{(1)}} \sin \left(\Phi_{c}+\theta_{1}^{(1)}-\theta_{1}^{(2)}\right)\right. \\
& \left.+\frac{\varepsilon_{i} A_{\mathrm{inj}}^{2}}{A_{1}^{(1)}} \sin \left(\Phi_{i}+\theta_{1}^{(1)}-2 \phi\right)\right] \\
\frac{d \theta_{1}^{(2)}}{d t}=\Delta \omega_{1}^{(2)}-\frac{\omega_{1}^{(2)}}{2 Q}\left[\frac{\varepsilon_{c} A_{1}^{(1)}}{A_{1}^{(2)}} \sin \left(\Phi_{c}+\theta_{1}^{(2)}-\theta_{1}^{(1)}\right)\right. & \\
& +\frac{\varepsilon_{c} A_{2}^{(1)}}{A_{1}^{(2)}} \sin \left(\Phi_{c}+\theta_{1}^{(2)}-\theta_{2}^{(1)}\right) \\
& \left.+\frac{\varepsilon_{i} A_{\text {inj }}^{2}}{A_{1}^{(2)}} \sin \left(\Phi_{i}+\theta_{1}^{(2)}-2 \phi+\pi\right)\right] \\
\frac{d \theta_{2}^{(1)}}{d t}=\Delta \omega_{2}^{(1)}-\frac{\omega_{2}^{(1)}}{2 Q}\left[\frac{\varepsilon_{c} A_{1}^{(2)}}{A_{2}^{(1)}} \sin \left(\Phi_{c}+\theta_{2}^{(1)}-\theta_{1}^{(2)}\right)\right. & +\frac{\varepsilon_{c} A_{2}^{(2)}}{A_{2}^{(1)}} \sin \left(\Phi_{c}+\theta_{2}^{(1)}-\theta_{2}^{(2)}\right) \\
& \left.+\frac{\varepsilon_{i} A_{\mathrm{inj}}^{2}}{A_{2}^{(1)}} \sin \left(\Phi_{i}+\theta_{2}^{(1)}-2 \phi+2 \xi\right)\right] \\
\frac{d \theta_{2}^{(2)}}{d t}=\Delta \omega_{2}^{(2)}-\frac{\omega_{2}^{(2)}}{2 Q}\left[\frac{\varepsilon_{c} A_{2}^{(1)}}{A_{2}^{(2)}} \sin \left(\Phi n_{c}+\theta_{2}^{(2)}-\theta_{2}^{(1)}\right)\right. & \left.\frac{\varepsilon_{i} A_{\mathrm{inj}}^{2}}{A_{2}^{(2)}} \sin \left(\Phi_{i}+\theta_{2}^{(2)}-2 \phi+2 \xi+\pi\right)\right] .
\end{aligned}
$$




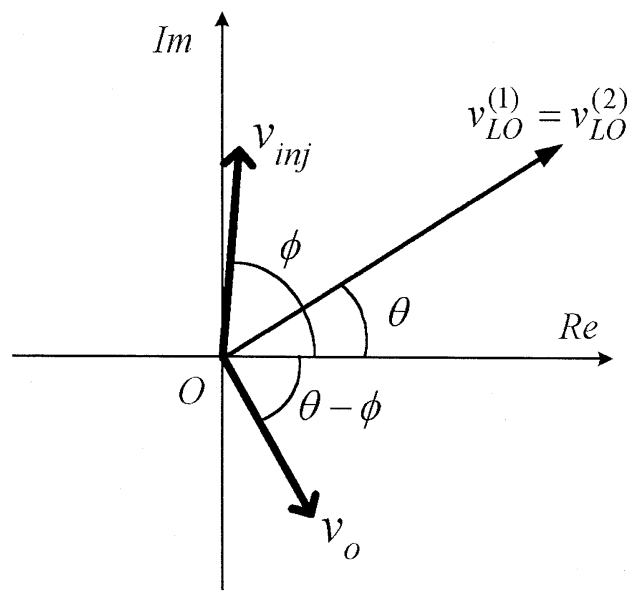

(a)

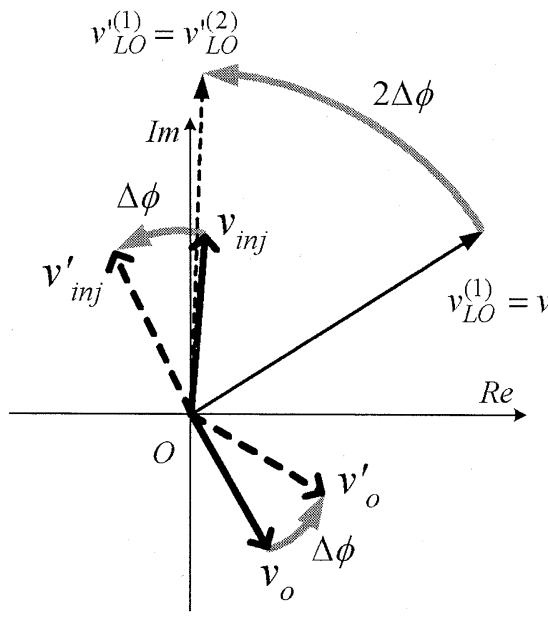

(b)

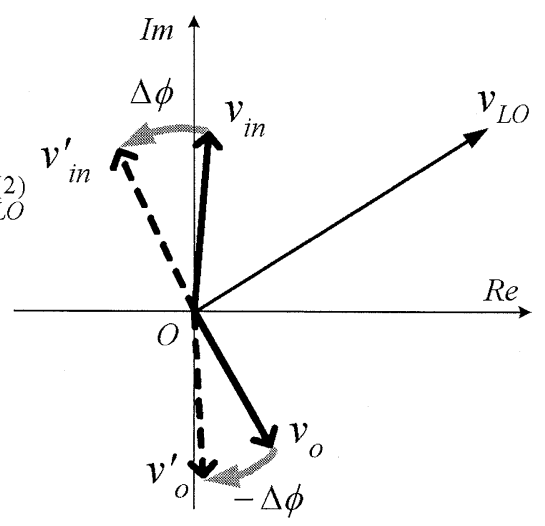

(c)

Fig. 4. Phasor diagrams of (a) the phase conjugation circuit under ideal situation, (b) the results as a phase shift $\Delta \phi$ in injection signal $\nu_{\text {inj }}$, and (c) conventional heterodyne circuit for comparison.

$\varepsilon_{c}$ and $\Phi_{c}$ are the coupling coefficient and coupling phase between SILSOMs. $\Phi_{c}$ is designed to be zero for the in-phase coupling. $\varepsilon_{i}$ and $\Phi_{i}$ are the coupling coefficient and the coupling phase of the subharmonic injection signals and the SILSOMs. The subscripts specify the elements of the antenna array, while the superscripts specify the two SILSOMs in each antenna element as shown in Fig. 3. All four oscillating phases are shown affected by the adjacent oscillating signals and the subharmonic injection signals. Taking (15a) as an example, the first term in the square bracket is the contribution of the coupling signal from $\nu_{L O, 1}^{(2)}$, which is at the fundamental frequency. The second term in the square bracket is the contribution of the subharmonic injection signal, which is now expressed as the square of $\nu_{\mathrm{inj}, 1}$ due to the second-harmonic relation. The frequency detuning is $\Delta \omega_{1}^{(1)}=\omega_{o, 1}^{(1)}-2 \omega_{\text {inj. }}$

Assuming the amplitudes and free-running frequencies of all four oscillators are the same, one can numerically solve the four mutually coupled nonlinear (15) and substitute the results into (6) and (10a). The output signals of the phase conjugation circuit are given as

$$
\nu_{o, 1}=c_{2} A_{\mathrm{inj}} A_{\mathrm{LO}} e^{j(\omega t / 2-\phi)} \frac{e^{j \theta_{1}^{(1)}}+e^{j \theta_{1}^{(2)}}}{2}+\text { h.o.t. }
$$

$$
\nu_{o, 2}=c_{2} A_{\mathrm{inj}} A_{\mathrm{LO}} e^{j(\omega t / 2-\phi+\xi)} \frac{e^{j \theta_{2}^{(1)}}+e^{j \theta_{2}^{(2)}}}{2}+\text { h.o.t. }
$$

Here, the phase distortion is assumed to be insignificant so that the first-order term is negligible. Under this assumption, $\left(e^{j \theta_{1}^{(1)}}+e^{j \theta_{1}^{(2)}}\right) / 2$ is approximately equal to $e^{j\left(\theta_{1}^{(1)}+\theta_{1}^{(2)}\right) / 2}$. Hence, (16) becomes

$$
\begin{aligned}
& \nu_{o, 1}=c_{2} A_{\mathrm{inj}} A_{\mathrm{LO}} e^{j\left[\omega t / 2-\phi+\left(\theta_{1}^{(1)}+\theta_{1}^{(2)}\right) / 2\right]} \\
& \nu_{o, 2}=c_{2} A_{\mathrm{inj}} A_{\mathrm{LO}} e^{j\left[\omega t / 2-\phi+\left(\theta_{2}^{(1)}+\theta_{2}^{(2)}\right) / 2+\xi\right]} .
\end{aligned}
$$

The phasor diagrams to illustrate the signals in the phase conjugation circuit consisting of two SILSOMs under the ideal situation are shown in Fig. 4. Fig. 4(a) shows the phasor diagram of the injection signal $\nu_{\text {inj }}$, LO signals $\nu_{\mathrm{LO}}^{(1)}$ and $\nu_{\mathrm{LO}}^{(2)}$, and output signal $\nu_{o}$ based on (6)-(11). Note the subharmonic and the fundamental signals are operated at different frequencies; hence their phasors shown in the same diagram are based on this understanding and made for illustration purposes. Fig. 4(b) shows that a phase shift $\Delta \phi$ in the subharmonic injection signal $\nu_{\text {inj }}^{\prime}$ gives a phase shift $2 \Delta \phi$ in the LO signals $\nu_{\mathrm{LO}}^{\prime(1)}$ and $\nu_{\mathrm{LO}}^{\prime(2)}$ due to the locking phenomenon. Consequently, the phase shift in the output signal $\nu_{o}^{\prime}$ at the subharmonic frequency is $\Delta \phi$, which is the same as the injection signal. In other words, the 


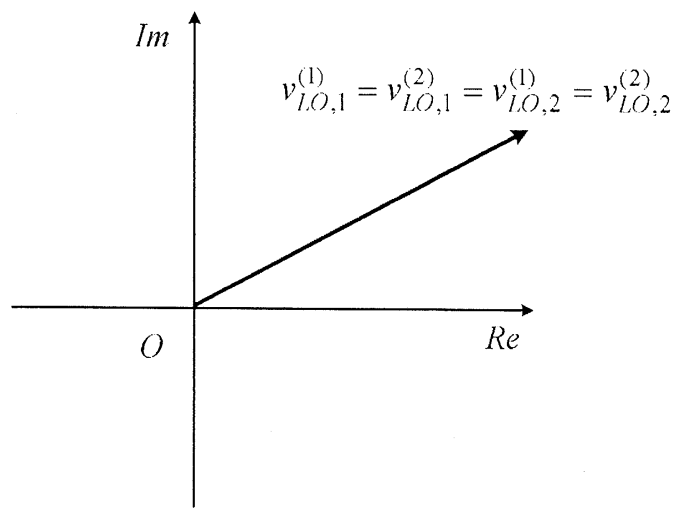

(a)

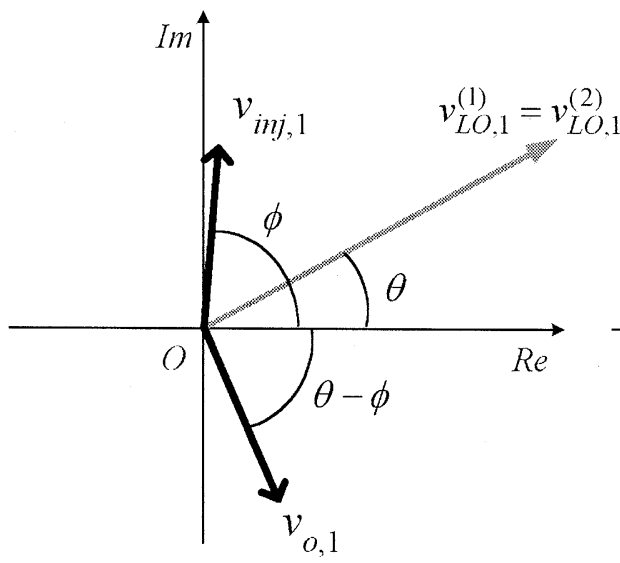

(b)

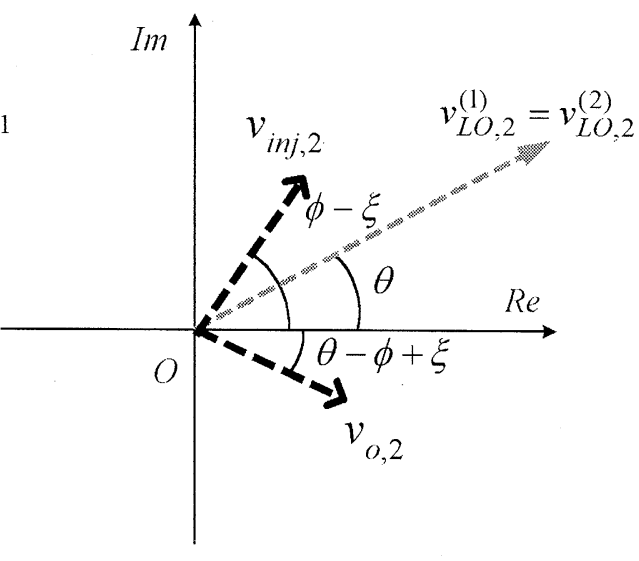

(c)

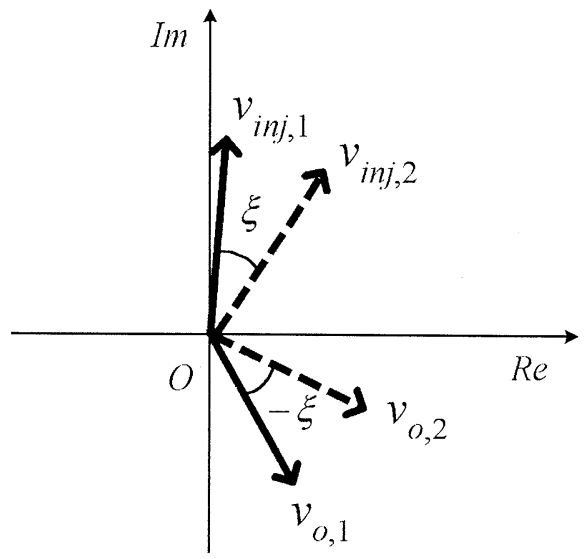

(d)

Fig. 5. Phasor diagrams of a two-element phase conjugation circuit under ideal situation. (a) Four in-phase LO signals. The mixing operations in (b) the first element, (c) the second element, and (d) the resulting output signals described by (12).

frequency and phase variation of the output signal follow the incident signal for the SILSOM operation. In the conventional heterodyne circuit given in Fig. 1, the phase shift $\Delta \phi$ in the input signal, however, has no effect on the LO signal $\nu_{\mathrm{LO}}$. The phase shift in the output signal is then $-\Delta \phi$, as shown in Fig. 4(c). This indicates that the frequency and phase variation of the output signal change oppositely to those of the incident signal as for a conventional mixer operation.

Figs. 5 and 6 further illustrate the phasor diagrams of a two-element phase conjugation circuit under ideal and practical situations. Fig. 5(a) shows the signals of four in-phase locked oscilla- tors given by (6). The mixing operations in the first and second element are shown in Fig. 5(b) and (c), respectively. $\xi$ is the phase difference of two incident signals. The resulting phase difference of the two output signals becomes $-\xi$, as shown in Fig. 5(d), which is in consistence with (12).

Fig. 6(a) shows the four non-in-phase oscillating signals given by (15). The LO signals of the first and second elements shown in Fig. 6(b) and (c) are slightly different in phase. In consequence, the phase difference of the output signals becomes $\left(\theta_{1}^{(1)}+\theta_{1}^{(2)}-\theta_{2}^{(1)}-\theta_{2}^{(2)}\right) / 2-\xi$, as shown in Fig. 6(d), rather than $-\xi$, given in Fig. 5(d). In other words, 


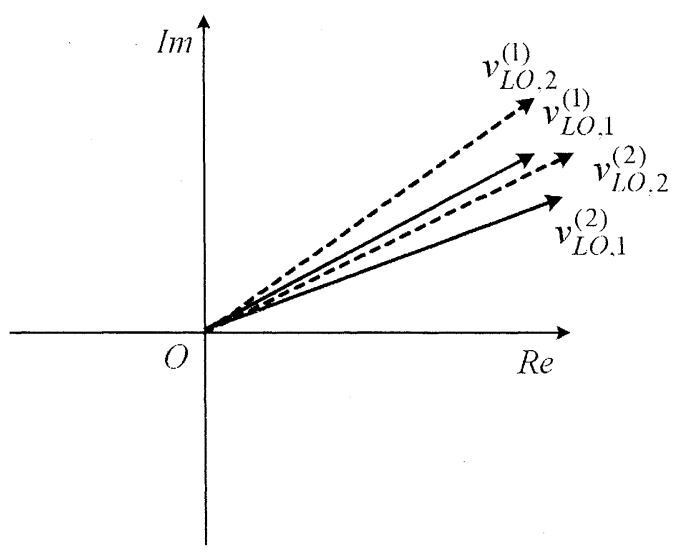

(a)

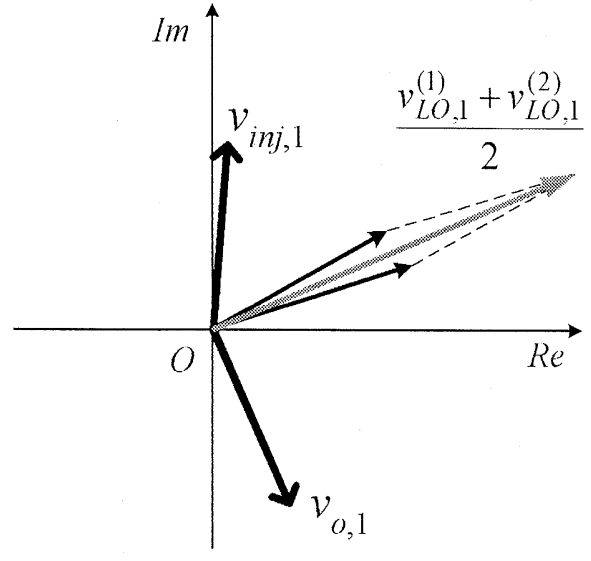

(b)

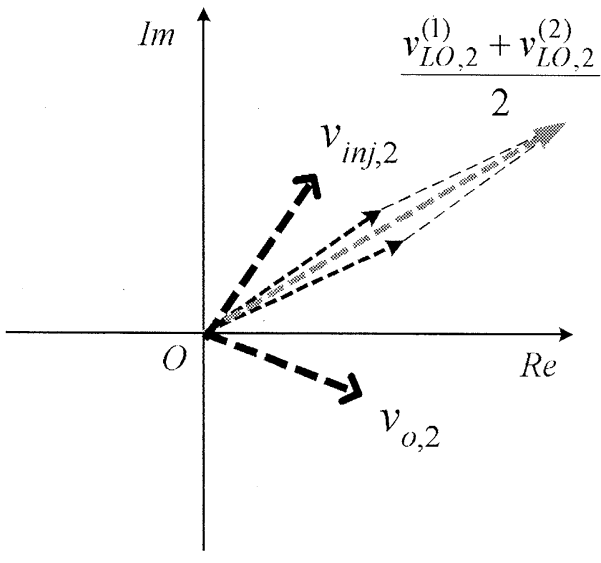

(c)

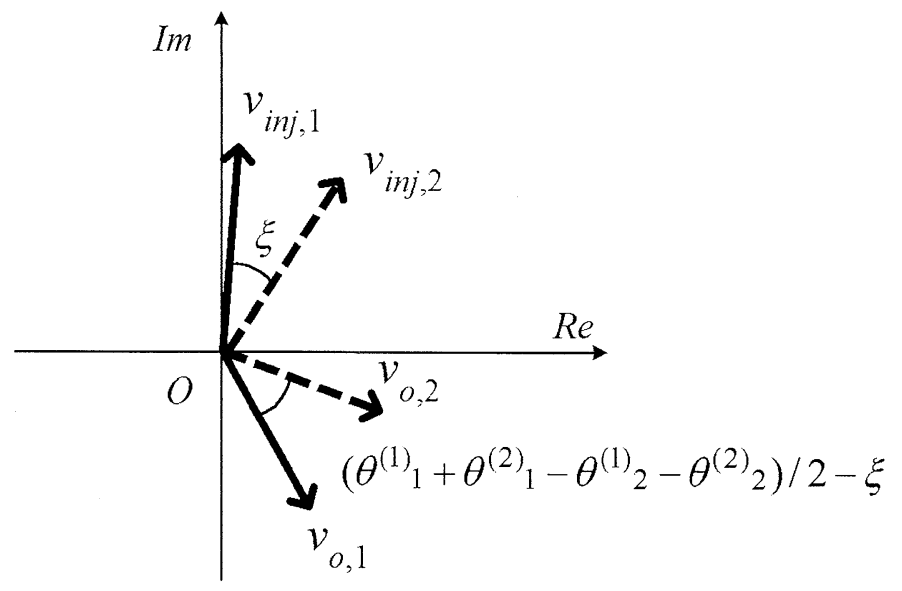

(d)

Fig. 6. Phasor diagrams of a two-element phase conjugation circuit under practical consideration. (a) Four non-in-phase LO signals. Mixing operations in (b) the first element, (c) the second element, and (d) the resulting output signals described by (14)-(17).

when the four phase terms $\theta_{1}^{(1)}, \theta_{1}^{(2)}, \theta_{2}^{(1)}$, and $\theta_{2}^{(2)}$ are not identical, the phase distortion in the output signals will be observed. In addition, for the phase conjugation operation shown in Figs. 4-6, since the LO signals are locked to the injection signals, the phase distribution among the output signals is in a reversed order as the injection signals in the space domain, but the phase variation in time domain is preserved. For the conventional heterodyne array, both the phase distribution in space domain and phase variation in time domain are reversed by using external LO signals.

\section{CiRCUit IMPLEMENTATION}

This section is the detailed description of the circuit design of a two-element retro-directive antenna array. More elements can be added to be a 1-D retro-directive antenna array as shown in Fig. 3.

\section{A. Phase Conjugation Circuit}

Fig. 7 shows the circuit schematic diagram of a two-element phase conjugation circuit using NE32684A high electron 


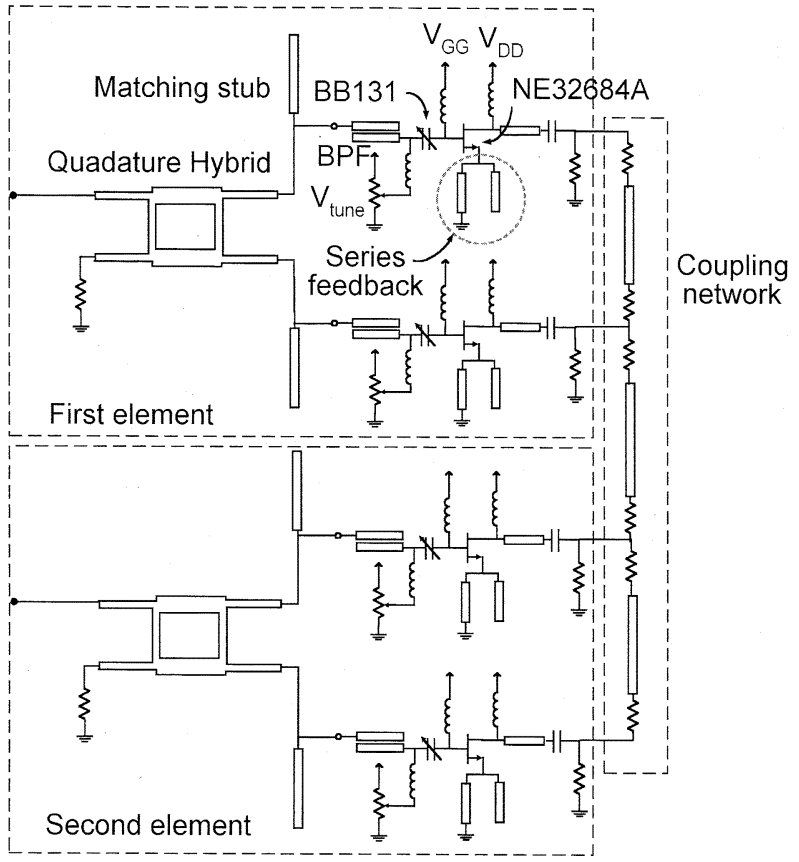

Fig. 7. Circuit schematic diagram of a two-element phase conjugation circuit.

mobility transistor (HEMT) devices. The series feedback at source terminal is to provide negative resistance [15]. The short stub gives a de return path, and the open stub makes the source terminal in series with a desired impedance to allow the transistor unstable near the designed frequency. A quarter-wavelength coupler at the gate terminal is a dc-blocking element as well as a bandpass filter to filter out the LO signal. The tuning varactor diode is Philips BB131. The SILSOM signal is designed to oscillate at $5.8 \mathrm{GHz}$ with the subharmonic injection signal at $2.9 \mathrm{GHz}$.

To enhance the second-order nonlinear term of the SILSOM output signal and maintain enough gain of the HEMT for oscillation, optimum values of bias voltages $V_{G G}$ and $V_{D D}$ are selected to give a maximum conversion gain $\left|c_{2} A_{\mathrm{LO}}\right|$ In the measurement, the SILSOMs are biased at $V_{G G}=-0.3 \mathrm{~V}$ and $V_{D D}=2 \mathrm{~V}$, and the oscillator free-running frequency is tuned by $\nu_{\text {tune }}$ through a variable resistor. The in-phase coupling network is constructed by transmission lines loaded with resistors [16], [17]. The input and output matching circuit are designed with open stubs at the subharmonic frequency. The $90^{\circ}$ branch line couplers are used to separate the first-order terms and the second-order terms of the SILSOM output signals based on (11).

Fig. 8(a) shows the developed two circuit boards of the phase conjugation circuit using 1-mm-thick FR4 substrate. The left side is the radio-frequency (RF) unit and the right side is the dc bias and control unit.

\section{B. Retro-Directive Antenna Array}

In the implementation of the retro-directive antenna, proper amplifications of the received signals and retransmitted signals are considered in order to lock the SILSOMs and compensate the conversion loss of the phase conjugation circuit. Half-wavelength patch antennas loaded with two-stage amplifiers are de-

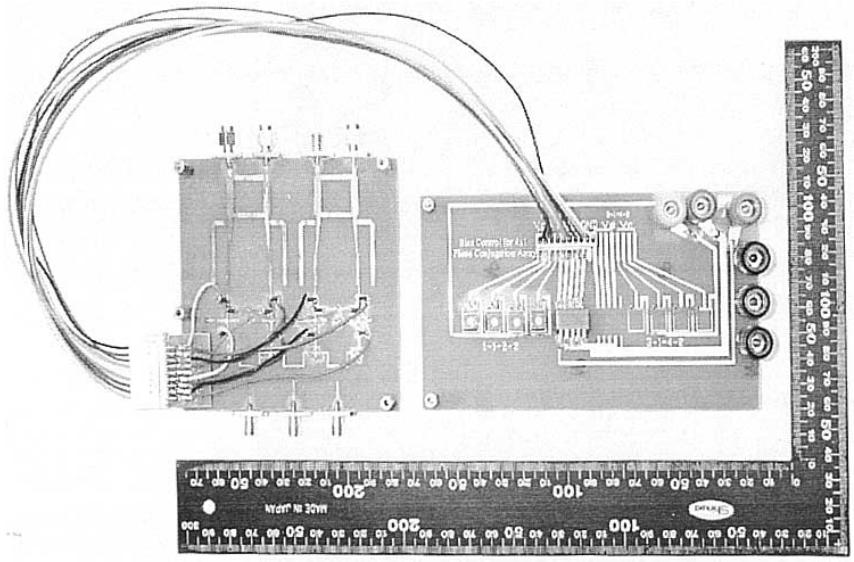

(a)

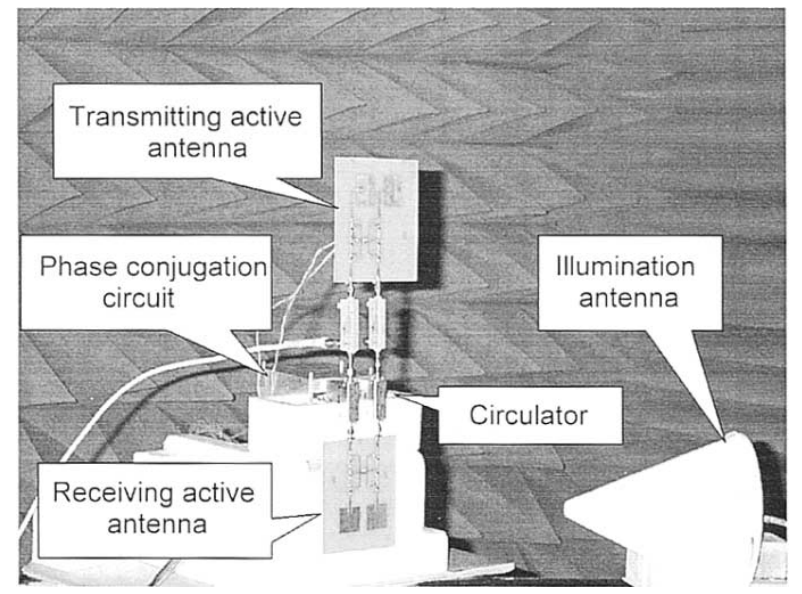

(b)

Fig. 8. Photographs of a two-element (a) phase conjugation circuit and (b) retro-directive antenna array inside an anechoic chamber.

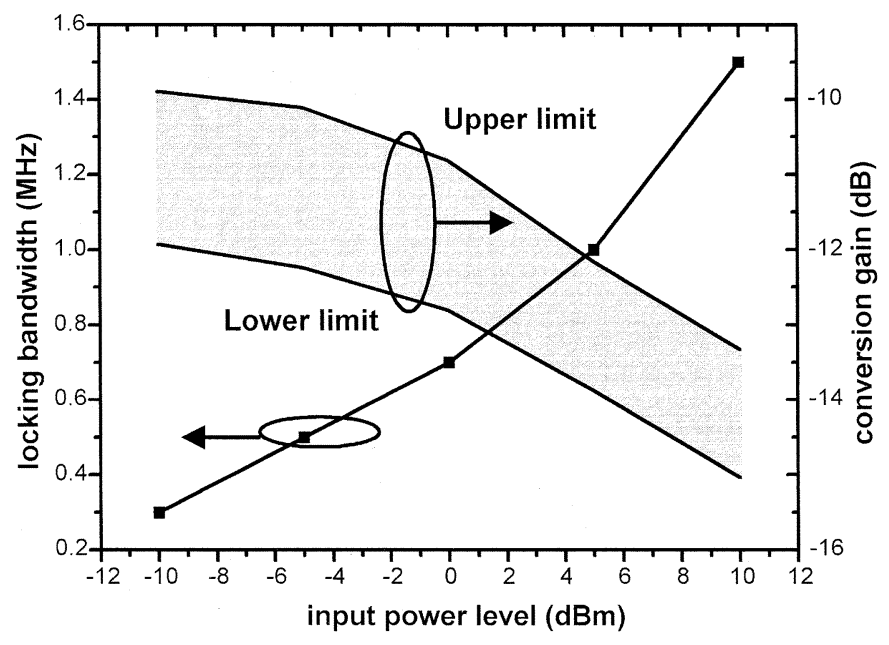

Fig. 9. Measured results of the locking bandwidth and conversion gain of the two-element phase conjugation circuit.

signed not only to provide about $15 \mathrm{~dB}$ power gain but also to increase the isolation between input and output signals. The transmitting and receiving antennas are in an orthogonal-polarized arrangement with about $15 \mathrm{~dB}$ isolation. The patch antennas are 


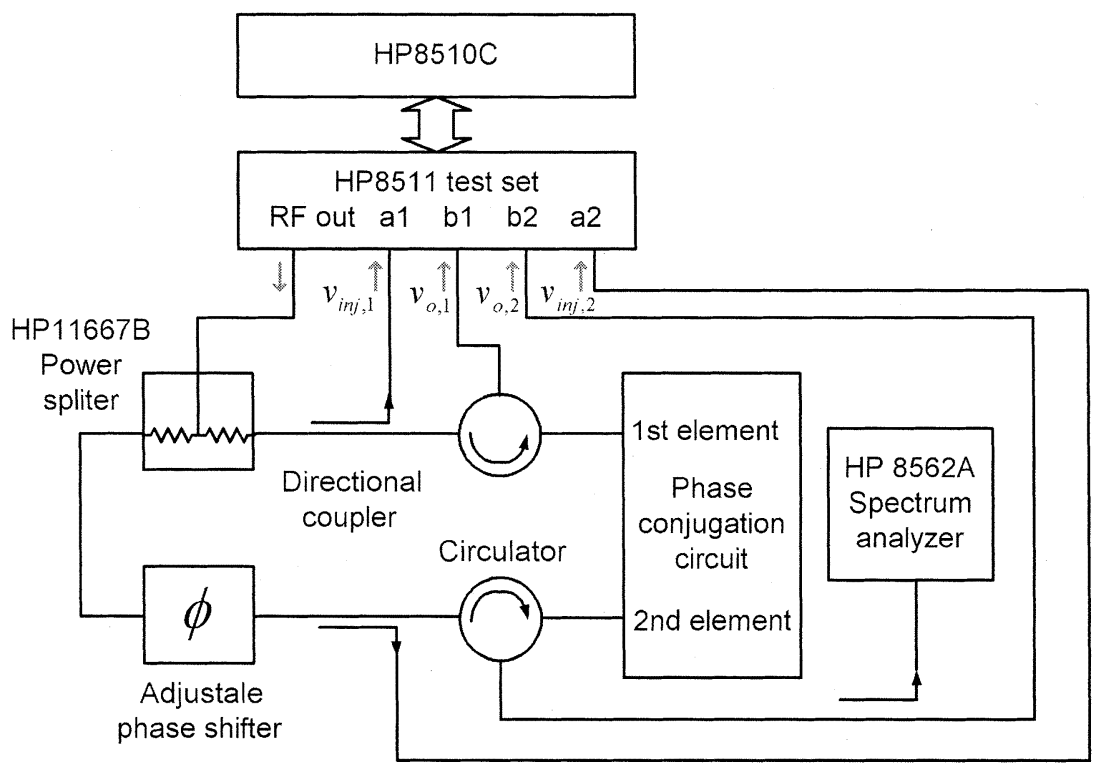

Fig. 10. Experimental arrangement for the phase conjugation circuit measurement.

fabricated on 1.6-mm-thick FR4 substrate with 0.4-wavelength spacing. Fig. 8(b) shows the pictorial view of the two-element retro-directive antenna array inside the anechoic chamber. Two DiTom D3C2040 circulators are used to separate the transmitting and receiving signal paths.

\section{RESULTS}

\section{A. Phase Conjugation Circuit}

The measurement results of locking range and conversion gain of the two-element phase conjugation circuit with different input power levels are shown in Fig. 9. Since the LO signal level varies slightly within the locking bandwidth, the conversion gain varies in pace with the LO signal level. The gray area is the region of the measured conversion gain within the locking range. It shows that as the input power level increases, the locking bandwidth increases and the conversion gain decreases.

The experimental arrangement to measure the phase relation of the input and output signals of the two-element phase conjugation circuit is shown in Fig. 10. An HP8510C vector network analyzer with HP8511 four-port test set is used to measure the four corresponding input and output signals for each element. In the measurement, an HP11667B 3-dB power splitter equally divides the RF out signal to the two-element phase conjugation circuit. An adjustable phase shifter is used to give different phase differences for the two input signals. The input signals are directed to the $a_{1}$ port and $a_{2}$ port of the HP8511 through directional couplers. The output signals from each element are then connected to the $b_{1}$ port and $b_{2}$ port. An HP8562A spectrum analyzer is used to monitor the locking characteristics of the output signal from the SILSOMs.

The calculated and measured results of the phase relation of the input and output signals are shown in Fig. 11. The simulation results are calculated based on (14)-(17). The coefficients in each equation of (15), for example, $\omega_{1}^{(1)} \varepsilon_{c} A_{1}^{(2)} / 2 Q A_{1}^{(1)}$, are determined from the measured locking bandwidth for each associated coupling. It shows that at the low injection signal power level, the input and output phase differences give a quasi-linear relation with the slope about -1 , as shown in Fig. 11(a). This closely corresponds to the ideal situation of phase conjugation as illustrated in Fig. 5. Note that the two gaps in the measured results or the slightly curved lines in the calculated results shown in the regions around $-90^{\circ}$ and $90^{\circ}$ are due to the unstable locking of the oscillators at these phase angles.

As the injection signal power level increases, the nonlinear effects on phase distortion are observed as shown in Fig. 11(b). The explanation of this phenomenon was illustrated in Fig. 6. As the injection power level increases further, the assumption of insignificant distortion is not satisfied and the first-order and higher order terms in (16) are not negligible. In addition, the saturation of the second-order term may give the harmonic relation in (15) differently. These then lead to more prominent discrepancy between the calculated and measured results, as shown in Fig. 11(c).

Figs. 9 and 11 show that the locking range becomes wider by increasing the input power level; however, the conversion loss and phase distortion are deteriorated. There is then a tradeoff between these parameters, which are described by (14)-(17).

\section{B. Retro-Directive Antenna Array}

To measure the bistatic retransmitted radiation characteristics of the developed retro-directive antenna array, the experimental arrangement is shown in Fig. 12. The incident wave comes from a vertically polarized illumination antenna at a fixed incident angle. The two-element retro-directive antenna array is located on a computer-controlled positioner. Its retransmitted radiation pattern is then measured by a horizontally polarized measurement antenna in the far field.

In the measurement arrangement, both the illumination and measurement antennas are TECOM 201085 dual-polarized an- 


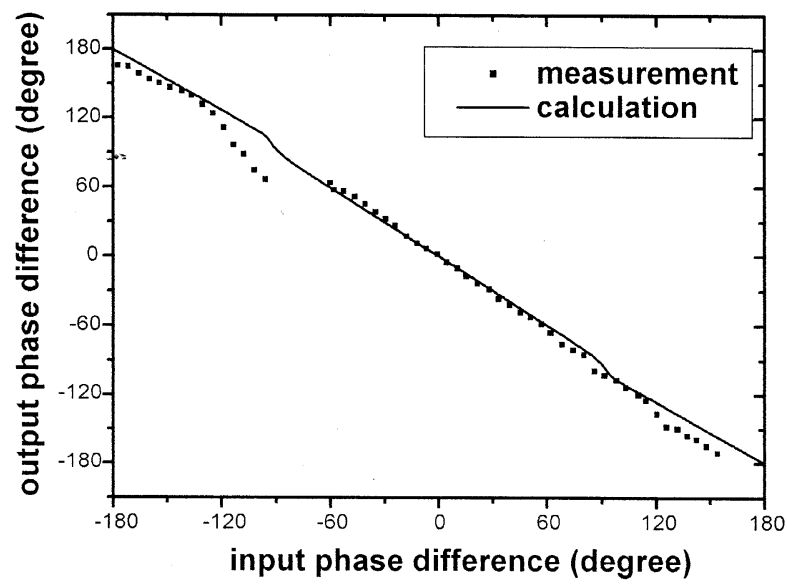

(a)

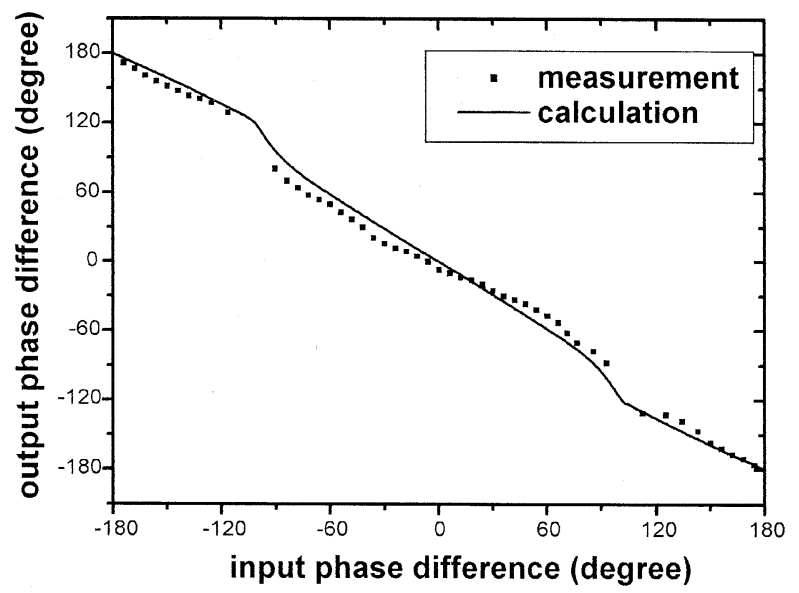

(b)

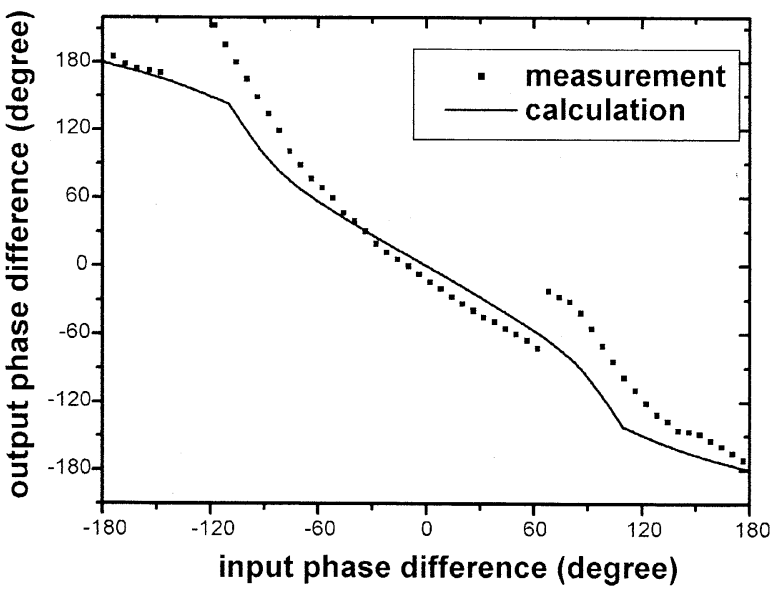

(c)

Fig. 11. Measured and calculated results of the phase relation for the input and output signals with input power levels at (a) -10 , (b) -5 , and (c) $0 \mathrm{dBm}$.

tennas with the unused ports terminated. The illumination antenna is about $40 \mathrm{~cm}$ from the test retro-directive antenna array, whereas the measurement antenna is at about $6 \mathrm{~m}$ in the far field. The illumination antenna is located on a plexiglass arm, which is attached on the rotating positioner to give a fixed incident angle $\theta_{i}$ during the measurement. A pictorial view of the illumination

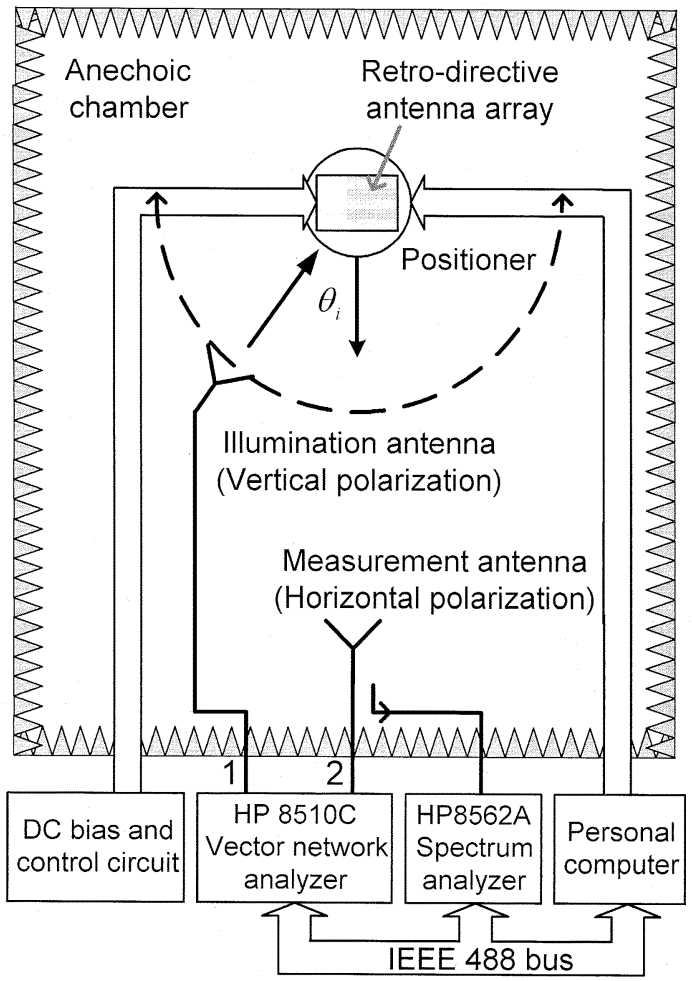

Fig. 12. Experimental arrangement for the retro-directive antenna array measurement.

antenna and the test retro-directive antenna array is shown in Fig. 8(b). A personal computer is adopted to control this measurement system via an IEEE-488 bus to perform positioner rotation, spectrum monitoring, and data acquisition.

The measured and calculated results at six different incident angles, from $-30^{\circ}$ to $45^{\circ}$ with $15^{\circ}$ spacing, are shown in Fig. 13. In each figure, the direction of the incident wave is indicated by an arrow. In the measurement, the responses of room clutter and the direct coupling between illumination and measurement antennas are subtracted in the calibration process. The calculated results of bistatic-retransmitted patterns are based on the calculated phase conjugation response given in (11). The measured results are shown in good agreement with the calculated results. The slight discrepancy in the radiation pattern near $\pm 90^{\circ}$ is from the finite ground plane of patch antenna.

\section{CONCLUSION}

In this paper, a novel circuit structure for phase conjugation is presented with analysis and numerical and experimental results. With the use of the balanced circuit structure of SILSOMs, no external LO signals are required and the output signals are operated at the same frequency of the input signals. In addition, the proposed phase conjugation circuit not only reverses the phase distribution in space domain but also preserves the frequency and phase variation in time domain. This characteristic makes it suitable for the frequency modulation and phase modulation operation. The developed phase conjugation circuit 


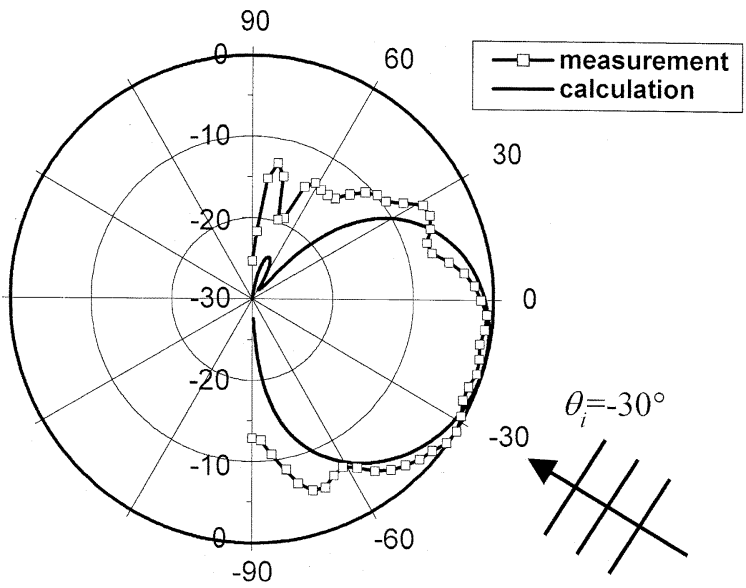

(a)

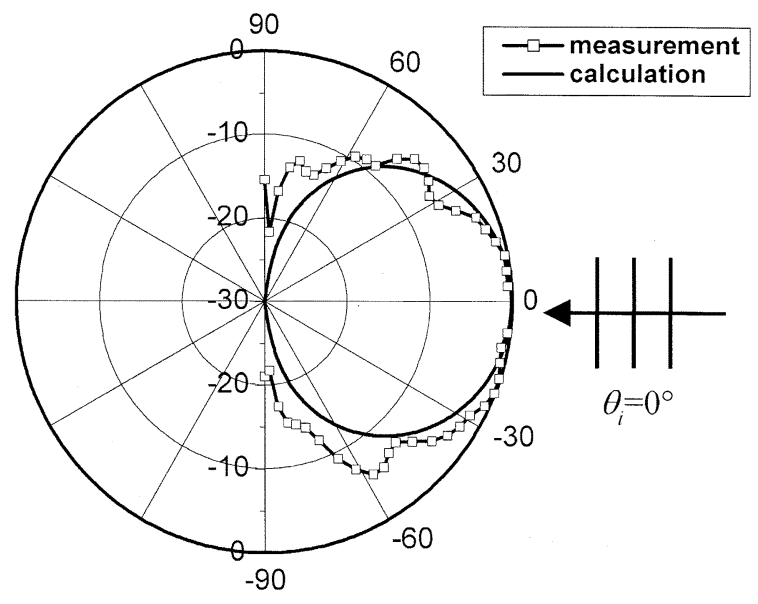

(c)

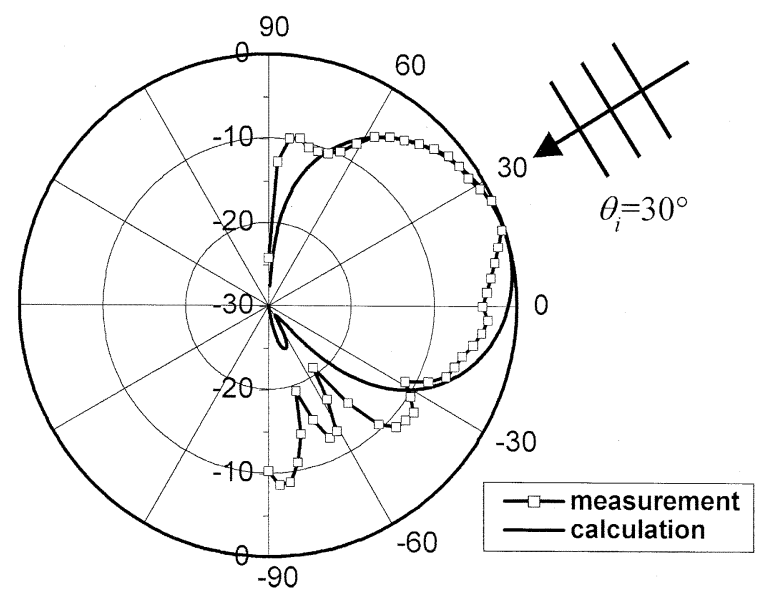

(e)

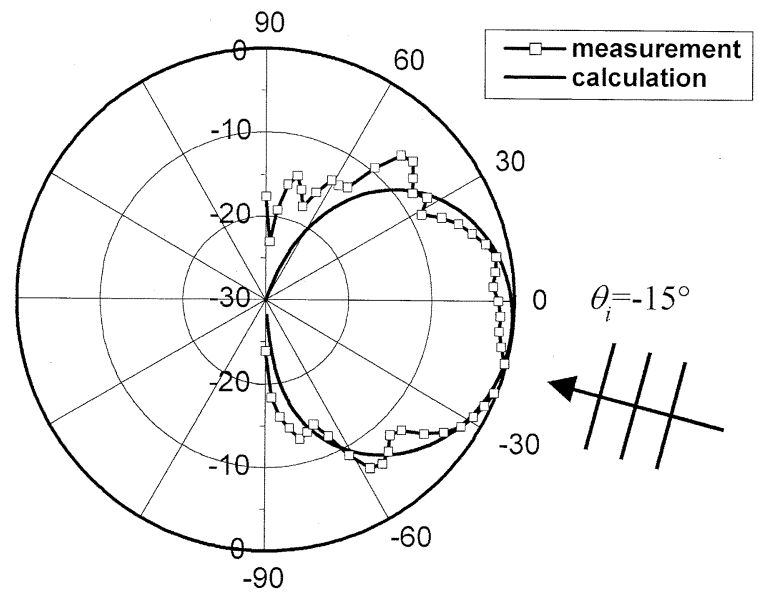

(b)

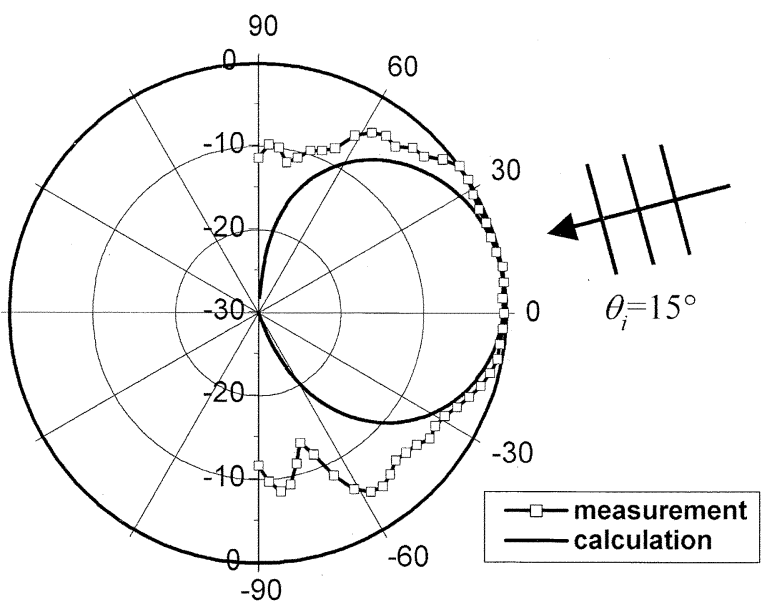

(d)

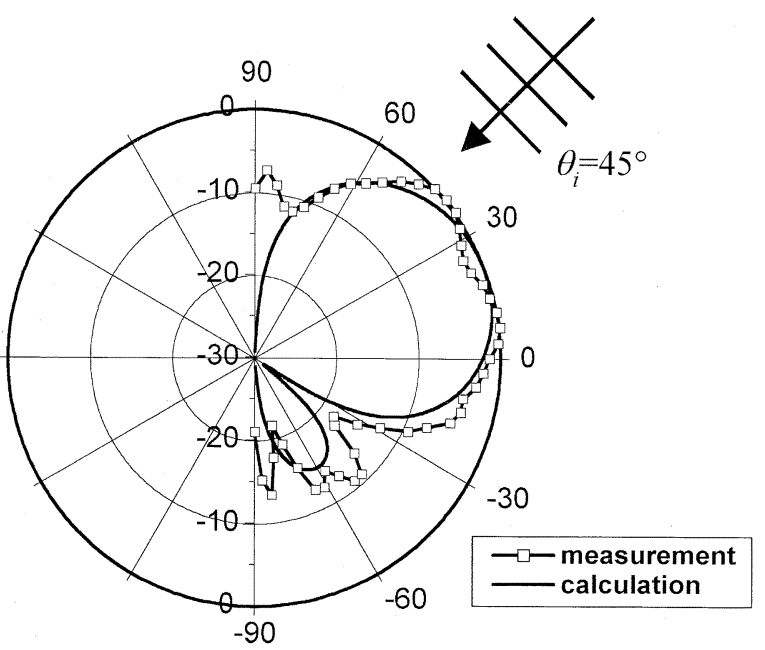

(f)

Fig. 13. Measured and calculated retransmitted radiation patterns of the retro-directive antenna with illumination antenna at the incident angles of (a) $-30^{\circ}$, (b) $15^{\circ}$, (c) $0^{\circ}$, (d) $15^{\circ}$, (e) $30^{\circ}$, and (f) $45^{\circ}$.

and retro-directive antenna array are measured and their performances shown in good agreement with the calculated results. The proposed retro-directive antenna array structure can be designed in a 2-D array for communication or tracking application by using a multilayer circuit structure.

\section{REFERENCES}

[1] M. Chryssomallis, "Smart antennas," IEEE Antennas Propagat. Mag., vol. 42, pp. 129-136, June 2000.

[2] Y. Zhou, P. C. Yip, and H. Leung, "Tracking the direction of arrival of multiple moving targets by passive array: Algorithm," IEEE Trans. Signal Processing, vol. 47, pp. 2655-2666, Oct. 1999. 
[3] B. S. Hewitt, "The evolution of radar technology into commercial systems," in Proc. IEEE MTT-S Int. Microwave Symp. Dig., vol. 2, May 1994, pp. 1271-1274.

[4] S. N. Andre and D. J. Leonard, "An active retrodirective array for satellite communications," IEEE Trans. Antennas Propagat., vol. AP-12, pp. 181-186, Feb. 1964.

[5] Y. Chang, H. R. Fetterman, L. Newberg, and S. K. Panaretos, "Microwave phase conjugation using antenna arrays," IEEE Trans. Microwave Theory Tech., vol. 46, pp. 1910-1919, Nov. 1998.

[6] S. L. Karode and V. F. Fusco, "Self-tracking duplex communication link using planar retrodirective antennas," IEEE Trans. Antennas Propagat., vol. 47, pp. 993-1000, June 1999.

[7] C. W. Pobanz and T. Itoh, "A microwave noncontact identification transponder using subharmonic interrogation," IEEE Trans. Antennas Propagat., vol. 43, pp. 1673-1679, July 1995.

[8] C. Y. Pon, "Retrodirective array using the heterodyne technique," IEEE Trans. Antennas Propagat., vol. AP-12, pp. 176-180, Feb. 1964.

[9] Y. R. Yang and T. H. Chu, "Locking performance analysis of MESFET subharmonically injection locked oscillators," IEEE Trans. Microwave Theory Tech., vol. 47, pp. 1014-1020, July 1999.

[10] S. C. Yen and T. H. Chu, "A retro-directive antenna array using phase conjugation circuit with subharmonically injection-locked self-oscillating mixer," in Proc. 32nd Eur. Microwave Conf., Sept. 2002, pp. 865-868.

[11] K. Kurokawa, "Injection locking of microwave solid-state oscillators," Proc. IEEE, vol. 61, pp. 1386-1410, Oct. 1973.

[12] R. Adler, "A study of locking phenomena in oscillators," Proc. IEEE, vol. 61, pp. 1380-1385, Oct. 1973.

[13] R. J. Pogorzelski, P. F. Maccarini, and R. A. York, "Continuum modeling of the dynamics of externally injection-locked coupled oscillator arrays," IEEE Trans. Microwave Theory Tech., vol. 47, pp. 471-478, Apr. 1999.

[14] J. A. Navarro and K. Chang, Integrated Active Antennas and Spatial Power Combining. New York: Wiley, 1996.

[15] G. Gonzalez and O. J. Sosa, "The design of a series-feedback network in a transistor negative-resistance oscillator," IEEE Trans. Microwave Theory Tech., vol. 47, pp. 42-47, Jan. 1999.
[16] R. A. York, P. Liao, and J. J. Lynch, "Oscillator array dynamics with broadband $n$-port coupling networks," IEEE Trans. Microwave Theory Tech., vol. 42, pp. 2040-2045, Nov. 1994.

[17] P. Liao and R. A. York, "A six-element beam-scanning array," IEEE Microwave Guided Wave Lett., vol. 4, pp. 20-22, Apr. 1994.

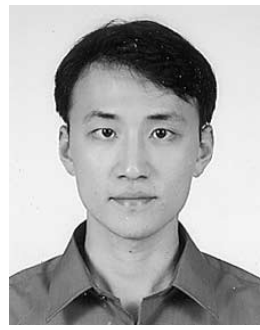

Shih-Chieh Yen (S'00) received the B.S. and M.S. degrees in electrical engineering from National Taiwan University, Taipei, Taiwan, R.O.C., in 1995 and 1997, respectively, where he is currently working toward the Ph.D. degree.

From 1997 to 1999, he served in the Chinese Army and was responsible for the maintenance of a wireless communication network. His research interests include linear and nonlinear microwave circuit design and analysis, microwave measurement, and active antenna array techniques.

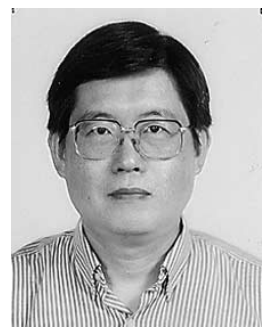

Tah-Hsiung Chu (M'87) received the B.S. degree from National Taiwan University, Taipei, Taiwan, R.O.C., in 1976 and the M.S. and Ph.D. degrees from the University of Pennsylvania, Philadelphia, in 1980 and 1983, respectively, all in electrical engineering.

From 1983 to 1986 , he was a Member of Technical Staff with the Microwave Technology Center, RCA David Sarnoff Research Center, Princeton, NJ. Since 1986, he has been on the Faculty of the Department of Electrical Engineering, National Taiwan University, where he is now a Professor of electrical engineering. His research interests include microwave-imaging systems and techniques, microwave circuits and subsystems, microwave measurements, and calibration techniques. 